University of Nebraska - Lincoln

DigitalCommons@University of Nebraska - Lincoln

March 1972

\title{
Potential-Barrier Effects in Photoabsorption. I. General Theory
}

Anthony F. Starace

University of Nebraska-Lincoln, astarace1@unl.edu

Follow this and additional works at: https://digitalcommons.unl.edu/physicsstarace

Part of the Physics Commons

Starace, Anthony F., "Potential-Barrier Effects in Photoabsorption. I. General Theory" (1972). Anthony F. Starace Publications. 16.

https://digitalcommons.unl.edu/physicsstarace/16

This Article is brought to you for free and open access by the Research Papers in Physics and Astronomy at DigitalCommons@University of Nebraska - Lincoln. It has been accepted for inclusion in Anthony F. Starace Publications by an authorized administrator of DigitalCommons@University of Nebraska - Lincoln. 


\title{
Potential-Barrier Effects in Photoabsorption. I. General Theory ${ }^{* \dagger}$
}

\author{
Anthony F. Starace $\$$ \\ Department of Physics, The Univer sity of Chicago, Chicago, Illinois 60637 \\ (Received 28 June 1971)
}

\begin{abstract}
This paper combines parts of the theories of spectroscopy, collisions, and autoionization to provide a unified framework for a quantitative theory of experimental data on $4 d$-shell photoabsorption in rare earths. The calculations by atomic theory are intended for tentative application to solids. The theory should also apply to $3 p$-shell photoabsorption in the transition metals and to other cases where the effect of a potential barrier is crucial. A prescription is given for the $a b$ initio numerical calculation of total and partial photoabsorption cross sections, as well as of the profiles, energies, and widths of resonances. A sum rule is derived for transitions of the type $l_{i}^{4 l_{i}+2} l_{f}^{N} \rightarrow l_{i}^{4 l_{i}+1} l_{f}^{N+1}$, which yields the integrated strength of observed absorption spectra.
\end{abstract}

\section{INTRODUCTION}

Recent experiments ${ }^{1,2}$ on the $4 d$-shell photoabsorption spectra of the (trivalent) rare earths show a prominent peak about 6-18 eV above threshold. In many of the rare earths this main peak appears to be composed of several peaks that are not completely resolved. The magnitude of this main structure varies greatly: Its experimental width at halfmaximum varies between 6 and $26 \mathrm{eV}$, and its strength decreases to zero with increasing atomic number $(57 \leqslant Z \leqslant 71$ ), i. e., as the $4 f$ subshell fills. Also present in the absorption spectra are numerous weak lines in the vicinity of the $4 d$-shell threshold. At lutetium $(Z=71)$ all these absorption features disappear.

These experiments have been interpreted in a preliminary report $t^{3}$ as being due to dipole transitions of the type $4 d^{10} 4 f^{N} \rightarrow 4 d^{9} 4 f^{N+1}$. The supporting arguments are these: (i) An independent-particle model $^{4}$ shows that at $\mathrm{La}(Z=57)$ the $4 f$ wave function becomes bound inside the centrifugal potential barrier and overlaps the $4 d$ wave function; higher-energy $f$ orbits remain outside the centrifugal barrier and have almost no overlap with the $4 d$ wave function. Hence, essentially all photoabsorption strength from the $4 d$ shell in the rare earths should go to the $4 f$ shell. (ii) The excellent overlap of the $4 f$ orbit with the $4 d$ orbit also implies a strong exchange interaction between the $4 f^{N+1}$ subshell configuration and the $4 d$ vacancy. This interaction is so strong that it splits the term levels of the $4 d^{9} 4 f^{N+1}$ configuration by about $20 \mathrm{eV}$, sending some levels far above threshold. These levels then autoionize to the various channels having the core configuration $4 d^{9} 4 f^{N}$. (iii) The observed decrease in strength of the main absorption peak with increasing atomic number (i.e., as the $4 f$ subshell fills) is understood in terms of the exclusion principle. A sum rule has been derived which shows that the summed line strength is proportional to the number of vacancies in the $4 f$ subshell. This sum rule is given in the Appendix of this paper. (iv) The discrete energy levels of the $4 d^{9} 4 f^{N+1}$ configuration were calculated by diagonalizing this configuration's interaction matrix using experimental spin-orbit parameters and multiplying the calculated Slater integrals by a single parameter which is adjusted to obtain a best fit to experimental levels. Agreement is excellent for those cases tried, namely, La, Ce, $\mathrm{Er}$, and Tm. Calculations for these and other rare earths will be reported in a detailed paper by Sugar. ${ }^{5}$

In this paper we present a comprehensive theory for the $4 d$-shell photoabsorption spectra observed in the rare earths. This theory combines elements from theoretical spectroscopy, collision theory, and the theory of autoionization; it pieces them together to provide a unified framework for the understanding of these new experimental phenomena. Our emphasis throughout is on providing the basis for future $a b$ initio numerical calculations of photoabsorption and photoionization intensities, including, specifically, the partial cross sections for ionization into particular final channels and the energies, widths, and line profiles of the $4 d^{9} 4 f^{N+1}$ resonances above threshold. In particular, we have enlarged the scope of the theory of Fano ${ }^{6}$ and Mies ${ }^{7}$ for multilevel multichannel autoionization by showing how to construct the prediagonalized states which these authors assume at the outset.

In the theoretical development that follows we consider photoabsorption by a single rare-earth atom, with the understanding that our results are meant to interpret the experimental spectra for rare-earth solids. This is justified because the outer electrons in the $n=5$ shell (i.e., $5 s^{2} 5 p^{6}$ ) screen the $4 f$ and lower subshells from the environment of the solid, or, in other words, the radius of the $4 f$ orbit is small compared to the radii of the $n=5$ orbits. $^{8}$ Since dipole absorption, exchange interaction, and autoionization all occur within the radius of the $n=4$ shell (i. e., the appropriate ma- 
trix elements all involve overlap with the $4 f$ wave function) it makes little difference whether one considers an atom in a gas or an ion in a metal. In particular, we ignore any modification of our atomic continuum wave functions arising from interactions specific to the solid state or to outer shell electrons.

Other important points that are implied in the rest of this paper are the following:

(i) The levels of $4 d^{9} 4 f^{N+1}$ are regarded as entirely separate from the Rydberg series $4 d^{9} 4 f^{N} n f$. Besides having different level structures, these two configurations differ in that the $4 f$ orbit is bound inside the potential barrier whereas the $n f$ orbit $(n \geqslant 5)$ is not. The final channels are thus considered to start with the $n=5$ configuration.

(ii) Dipole transitions $4 d \rightarrow p$ are not considered explicitly. They are allowed and should, in principle, be considered as additional final-state channels. However, preliminary numerical calculations ${ }^{9}$ indicate that the autoionizing levels decay 100 times more strongly to the $f$ channels than to the $p$ channels. Hence, for simplicity, we shall not mention the $p$ channels any further.

(iii) All interacting final states must have the same total angular momentum $J$ and projection $M_{J}$, but we do not single out $J$ and $M_{J}$ from the other quantum numbers that identify the final states.

(iv) Implied in our summations for total cross sections is a sum over all dipole-allowed total angular momentum quantum numbers $J$ and $M_{J}$.

(v) This paper considers only the final-state wave function. We do not consider the initial-state wave function explicitly since this constitutes a well-defined separate problem.

In Sec. II we indicate the procedure for obtaining the prediagonalized states of Fano $^{6}$ and Mies. ${ }^{7}$ In Sec. III we discuss the shift in the resonance energies due to interaction of the autoionizing levels with the continuum channels. In Sec. IV we obtain the final-state standing-wave eigenvector. In Sec. $\mathrm{V}$ we switch to incoming wave eigenvectors and derive expressions for partial and total cross sections, which show explicit resonance behavior. In Sec. VI we give a prescription for numerical calculations. Finally, in the Appendix we present a sum rule for the sum over all final levels of the line strength for transitions from a closed shell to a partially filled shell. The summed strength is found to be independent of the state of the initial configuration and proportional to the number of vacancies in the initial partially filled subshell.

\section{DIAGONALIZATION OF HAMILTONIAN SUBMATRICES}

We start from a zero-order basis set obtained from an independent-particle model such as Hartree-Fock or any of various central-field models. ${ }^{10}$ Using this basis set we construct linear combina- tions of Slater determinants for the relevant configurations, which we identify by the following notation:

$$
\begin{aligned}
& \Phi^{N+1} \equiv 4 d^{9} 4 f^{N+1}, \\
& \Phi^{N} \in f \equiv 4 d^{9} 4 f^{N} \in f .
\end{aligned}
$$

As discussed in the Introduction, other (usually closed) shells of electrons are implied in our notation, but not indicated explicitly, in order to emphasize the essential features of this problem. Note that we shall use lower-case Greek letters to denote both the coupling schemes and the quantum numbers necessary to specify uniquely the states of the above configurations. Upper-case Greek letters (other than $\Phi$ ) will be used to identify the various states pertaining to a wave function of energy $E$ that is not associated with any particular configuration [e.g., see Eq. (2) below]. The various states having the configurations (1) are thus denoted by $\left|\Phi^{N+1} \alpha\right\rangle$ and $\left|\Phi^{N} \lambda, \epsilon f, \rho\right\rangle$, where $\alpha$ identifies the state of $\Phi^{N+1}, \lambda$ identifies the state of $\Phi^{N}$, and $\rho$ identifies the state of $\Phi^{N} \lambda, \epsilon f$. We denote the Hamiltonian, including both electrostatic and spin-orbit interaction between and within the configurations $\Phi^{N+1}$ and $\Phi^{N} \in f$, as $H$ and the independent-particle-model Hamiltonian as $H_{\text {mod. }}$. We define the residual interaction as $V_{\text {res }} \equiv H-H_{\text {mod }}$. Each of the states $\left|\Phi^{N+1} \alpha\right\rangle$ and $\left|\Phi^{N} \lambda, \epsilon f, \rho\right\rangle$ is an eigenfunction of $H_{\bmod }$ whose energy depends only on the configuration.

We represent an exact standing-wave final-state eigenfunction of the Hamiltonian $H$ as

$$
\begin{aligned}
|\Lambda E\rangle \equiv \sum_{\alpha}\left|\Phi^{N+1} \alpha\right\rangle A_{\alpha \Lambda}(E) \\
\quad+\sum_{\lambda, \rho} \int d \epsilon\left|\Phi^{N} \lambda, \epsilon f, \rho\right\rangle B(\lambda, \epsilon, \rho ; \Lambda E) .
\end{aligned}
$$

The quantum number $\Lambda$ identifies a particular state of the wave function having eigenvalue $E$. Taking matrix elements of $H$ between the ket $|\Lambda E\rangle$ on the right-hand side and either $\left\langle\Phi^{N+1} \alpha^{\prime}\right|$ or $\left\langle\Phi^{N} \lambda^{\prime}, \epsilon^{\prime}, f, \rho^{\prime}\right|$ on the left-hand side, we obtain the following linear equations for the coefficients $A$ and $B$ :

$$
\begin{aligned}
& \sum_{\alpha}\left\langle\Phi^{N+1} \alpha^{\prime}|H| \Phi^{N+1} \alpha\right\rangle A_{\alpha \Lambda}(E) \\
& +\sum_{\lambda, \rho} \int d \epsilon\left\langle\Phi^{N+1} \alpha^{\prime}\left|V_{\text {res }}\right| \Phi^{N} \lambda, \epsilon f, \rho\right\rangle \\
& \quad \times B(\lambda, \epsilon, \rho ; \Lambda E)=E A_{\alpha \cdot \Lambda}(E), \\
& \sum_{\alpha}\left\langle\Phi^{N} \lambda^{\prime}, \epsilon^{\prime} f, \rho^{\prime}\left|V_{\text {res }}\right| \Phi^{N+1} \alpha\right\rangle A_{\alpha \Lambda}(E) \\
& +\sum_{\lambda, \rho}^{\sum} \int d \epsilon\left\langle\Phi^{N} \lambda^{\prime}, \epsilon^{\prime} f, \rho^{\prime}|H| \Phi^{N} \lambda, \epsilon f, \rho\right\rangle \\
& \quad \times B(\lambda, \epsilon, \rho ; \Lambda E)=E B\left(\lambda^{\prime}, \epsilon^{\prime}, \rho^{\prime} ; \Lambda E\right) .
\end{aligned}
$$

The solution of Eqs. (3) and (4) for the coefficients $A$ and $B$ constitutes the main task of this paper. In the remainder of this section we concern ourselves with the first step of the complete solution, namely, 
the diagonalization of the Hamiltonian submatrices

$$
\left\langle\Phi^{N+1} \alpha^{\prime}|H| \Phi^{N+1} \alpha\right\rangle
$$

and

$$
\left\langle\Phi^{N} \lambda^{\prime}, \epsilon^{\prime} f, \rho^{\prime}|H| \Phi^{N} \lambda, \epsilon f, \rho\right\rangle \text {. }
$$

The submatrix $\left\langle\Phi^{N+1} \alpha^{\prime}|H| \Phi^{N+1} \alpha\right\rangle$ is of finite dimension and is diagonalized by the methods of theoretical spectroscopy. ${ }^{5}$ We label the new states with the index $\beta$. In this new representation the Hamiltonian submatrix is

$$
\left\langle\Phi^{N+1} \beta^{\prime}|H| \Phi^{N+1} \beta\right\rangle=E_{\beta} \delta_{\beta \beta^{\prime}} .
$$

Note that the $E_{\beta}$ are not quite the same as the energies of the experimentally observed resonances. We shall find in Sec. III that the resonance energies differ from $E_{\beta}$ because of the interaction of the discrete states $\left|\Phi^{N+1} \beta\right\rangle$ with the continuum channels.

The diagonalization of the Hamiltonian submatrix

$$
\left\langle\Phi^{N} \lambda^{\prime}, \epsilon^{\prime} f, \rho^{\prime}|H| \Phi^{N} \lambda, \epsilon f, \rho\right\rangle
$$

requires a different treatment because of its dependence on the continuous variables $\epsilon^{\prime}$ and $\epsilon$. We treat the problem by splitting the residual interaction submatrix into two parts, namely, one part (denoted $V_{\mathrm{res}}^{\mathrm{core}}$ ) involving only interactions among the electrons in the core configuration $\Phi^{N}$, these interactions being diagonal in $\epsilon$ and $\rho$; the other part (denoted $V_{\text {res }}^{\text {ec }}$ ) involving only electron-core interactions between the excited electron and the ionic-core configuration. We may thus write

$$
\begin{aligned}
& \left\langle\Phi^{N} \lambda^{\prime}, \epsilon^{\prime} f, \rho^{\prime}|H| \Phi^{N} \lambda, \epsilon f, \rho\right\rangle \\
& =\left(E_{\lambda}+\epsilon\right) \delta_{\lambda^{\prime} \lambda} \delta\left(\epsilon^{\prime}-\epsilon\right) \delta_{\rho^{\prime} \rho} \\
& +\left\langle\Phi^{N} \lambda^{\prime}\left|V_{\mathrm{res}}^{\mathrm{core}}\right| \Phi^{N} \lambda\right\rangle \delta\left(\epsilon^{\prime}-\epsilon\right) \delta_{\rho^{\prime} \rho} \\
& \quad+\left\langle\Phi^{N} \lambda^{\prime}, \epsilon^{\prime} f, \rho^{\prime}\left|V_{\mathrm{res}}^{\mathrm{ec}}\right| \Phi^{N} \lambda, \epsilon f, \rho\right\rangle,
\end{aligned}
$$

where we have used the relation

$$
H_{\text {mod }}\left|\Phi^{N} \lambda, \epsilon f, \rho\right\rangle=\left(E_{\lambda}+\epsilon\right)\left|\Phi^{N} \lambda, \epsilon f, \rho\right\rangle \text {. }
$$

Note that the core energies $E_{\lambda}$ are degenerate in the zero-order model and hence the first task is to split these levels by diagonalizing the matrix $\left\langle\Phi^{N} \lambda^{\prime}\left|V_{\text {res }}^{\text {core }}\right| \Phi^{N} \lambda\right\rangle$. That is, one must obtain a new set of states identified by $\left|\Phi^{N} \mu, \epsilon f, \sigma\right\rangle$, such that

$$
\left\langle\Phi^{N} \mu^{\prime}, \epsilon^{\prime} f, \sigma^{\prime}\left|H_{\text {mod }}+V_{\text {res }}^{\text {core }}\right| \Phi^{N} \mu, \epsilon f, \sigma\right\rangle
$$

$$
=\left(E_{\mu}+\epsilon\right) \delta_{\mu^{\prime} \mu} \delta\left(\epsilon^{\prime}-\epsilon\right) \delta_{\sigma^{\prime} \sigma},
$$

where $E_{\mu}$ is the experimentally observable energy of the $\mu$ th level of the ionic core configuration $\Phi^{N} .{ }^{11}$ In this new representation the Hamiltonian submatrix becomes

$$
\begin{aligned}
\left\langle\Phi^{N} \mu^{\prime}, \epsilon^{\prime} f, \sigma^{\prime}|H|\right. & \left.\Phi^{N} \mu, \epsilon f, \sigma\right\rangle \\
=\left(E_{\mu}+\right. & \epsilon) \delta_{\mu^{\prime} \mu} \delta\left(\epsilon^{\prime}-\epsilon\right) \delta_{\sigma^{\prime} \sigma} \\
& +\left\langle\Phi^{N} \mu^{\prime}, \epsilon^{\prime} f, \sigma^{\prime}\left|V_{\text {res }}^{\mathrm{ec}}\right| \Phi^{N} \mu, \epsilon f, \sigma\right\rangle .
\end{aligned}
$$

Note that the matrix elements of $V_{\text {res }}^{\mathrm{ec}}$ represent the difference between the interaction of the excited electron with the ionic core and the interaction of the excited electron with the independent-particlemodel potential.

The eigenvectors which diagonalize Eq. (8) and satisfy the required boundary conditions asymptotically are known as the "incoming-wave" eigenvectors. The boundary conditions they satisfy are, alternatively, that the photoionized electron recede to infinity either in a specified direction or in a specified angular momentum state. These incoming-wave eigenvectors, however, satisfy an integral equation having a complex energy denominator, which is inconvenient for numerical calculations. Therefore, we consider initially the standing-wave eigenvectors of the Hamiltonian, which satisfy real equations, and postpone application of the appropriate boundary conditions.

A standing-wave eigenvector for the Hamiltonian submatrix in Eq. (8) may be represented as a linear combination of the basis states $\left|\Phi^{N} \mu, \epsilon f, \sigma\right\rangle$. In writing this linear combination we introduce two new sets of coefficients, discussed further below. One of these is represented by matrix elements of an operator $K(\mathcal{E})$, which is determined from collision theory. The other set, denoted by $U_{\mu, \sigma ; \Gamma}$ $\times(\mathcal{E}) \cos \pi \eta_{\Gamma}$, must be determined by a separate calculation. Note that because the standing-wave eigenvector is a linear combination of basis states $\left|\Phi^{N} \mu, \epsilon f, \sigma\right\rangle$ it is not identified by any particular core state $\mu$ or excited-electron energy $\epsilon$. Rather, we denote the standing wave having total energy $\mathcal{E}$ by $\left|\Phi^{N} e \Gamma \mathcal{E}\right\rangle$, where $\Phi^{N}$ indicates the core configuration, $e$ indicates that there is an excited electron, and $\Gamma$ is an index identifying a particular standingwave state having energy $\mathcal{E}$. We thus write the standing-wave eigenvector as ${ }^{12}$

$$
\begin{aligned}
\left|\Phi^{N} e \Gamma \mathcal{E}\right\rangle=\sum_{\mu, \sigma}\left\{\left|\Phi^{N} \mu, \epsilon f, \sigma\right\rangle_{\mathcal{E}}+\sum_{\mu^{\prime}, \sigma^{\prime}}\right. & \int d \epsilon^{\prime}\left|\Phi^{N} \mu^{\prime}, \epsilon^{\prime} f, \sigma^{\prime}\right\rangle \\
& \left.\times \frac{\rho}{\mathcal{E}-E_{\mu^{\prime}}-\epsilon^{\prime}}\left\langle\Phi^{N} \mu^{\prime}, \epsilon^{\prime} f, \sigma^{\prime}|K(\mathcal{E})| \Phi^{N} \mu, \epsilon f, \sigma\right\rangle_{\mathcal{E}}\right\} U_{\mu, \sigma ; \Gamma}(\mathcal{E}) \cos \pi \eta_{\Gamma} .
\end{aligned}
$$

The subscripts $\mathcal{E}$ below the unprimed basis states $\left|\Phi^{N} \mu, \epsilon f, \sigma\right\rangle$ imply that these states have total energy $\mathcal{E}$ (i.e., $E_{\mu}+\epsilon=\mathcal{E}$ ), whereas the primed states assume all possible energies. The symbol $\rho$ denotes the Cauchy principal value is to be taken in integrating over the singularity in the energy denominator.

The operator $K(\mathscr{E})$ is just the reaction matrix of collision theory. It is determined as the solution of the 
following integral equation:

$$
\begin{array}{r}
\left\langle\Phi^{N} \mu^{\prime}, \epsilon^{\prime} f, \sigma^{\prime}|K(\mathcal{E})| \Phi^{N} \mu, \epsilon f, \sigma\right\rangle=\left\langle\Phi^{N} \mu^{\prime}, \epsilon^{\prime} f, \sigma^{\prime}\left|V_{\mathrm{res}}^{\mathrm{ec}}\right| \Phi^{N} \mu, \epsilon f, \sigma\right\rangle+\sum_{\mu^{\prime \prime}, \sigma^{\prime \prime}} \int d \epsilon^{\prime \prime}\left\langle\Phi^{N} \mu^{\prime}, \epsilon^{\prime} f, \sigma^{\prime}\left|V_{\mathrm{res}}^{\mathrm{ec}}\right| \Phi^{N} \mu^{\prime \prime}, \epsilon^{\prime \prime} f, \sigma^{\prime \prime}\right\rangle \\
\times \frac{\boldsymbol{P}}{\mathcal{E}-E_{\mu^{\prime \prime}}-\epsilon^{\prime \prime}}\left\langle\Phi^{N} \mu^{\prime \prime}, \epsilon^{\prime \prime} f, \sigma^{\prime \prime}|K(\mathcal{E})| \Phi^{N} \mu, \epsilon f, \sigma\right\rangle .(10)
\end{array}
$$

Note that in both Eqs. (9) and (10) the integration over energy includes a summation over the discrete Rydberg series starting with $5 f$ (since the $4 f$ levels are being treated as resonances). The energy of the $5 f$ level is close to threshold and hence one may consider the discrete levels as an appendage to the continuum by normalizing the $5 f$ level per unit energy and integrating from the energy of $5 f .{ }^{13} \mathrm{~A}$ discussion of the numerical methods used to solve Eq. (10) in a one-channel case has been given elsewhere. ${ }^{14}$ It should be emphasized here, though, that the integration over the singularity poses no numerical problems.

The matrix $U_{\mu, \sigma ; \Gamma}(\mathcal{E})$ is an orthogonal transformation (whose rows are indexed by the pair of quantum numbers $\mu, \sigma)$ which diagonalizes a submatrix of $\left\langle\Phi^{N} \mu, \epsilon f, \sigma|K(\mathcal{E})| \Phi^{N} \mu^{\prime}, \epsilon^{\prime} f, \sigma^{\prime}\right\rangle$. This submatrix is constructed by selecting the basis states with total energy $\mathcal{E}$ (i.e., $E_{\mu}+\epsilon=E_{\mu}{ }^{\prime}+\epsilon^{\prime}=\mathcal{E}$ ). We denote this submatrix by $\{K(\mathscr{E})\}_{\mu, \sigma ; \mu^{\prime}, \sigma^{\prime}}$; it is known in collision theory as the reaction matrix "on the energy shell." The matrix $U_{\mu, \sigma ; \Gamma}(\mathcal{E})$ is thus the orthogonal transformation that diagonalizes $\{K(\mathcal{E})\}_{\mu, \sigma ; \mu^{\prime}, \sigma^{\prime}}$ and is obtained by solving the system of equations

$$
\begin{aligned}
\sum_{\mu^{\prime}, \sigma^{\prime}}\{K(\mathcal{E})\}_{\mu, \sigma ; \mu^{\prime}, \sigma^{\prime}} U_{\mu^{\prime}, \sigma^{\prime} ; \Gamma}(\mathcal{E}) \\
=-\pi^{-1}\left(\tan \pi \eta_{\Gamma}\right) U_{\mu, \sigma ; \Gamma}(\mathcal{E}),
\end{aligned}
$$

where $-\pi^{-1} \tan \pi \eta_{\Gamma}$ is the $\Gamma$ th eigenvalue of $\{K(\mathcal{E})\}$. Now the scattering matrix $S$ is related to the matrix $\{K(\mathcal{E})\}$ by the well-known formula ${ }^{12}$

$$
S(\mathcal{E})=\frac{1-i \pi\{K(\mathscr{E})\}}{1+i \pi\{K(\mathcal{E})\}} .
$$

Note that the $S$ matrix is also diagonalized by the orthogonal matrix $U$ and its $\Gamma$ th eigenvalue is $e^{i 2 \pi \eta} \Gamma$. Thus the label $\Gamma$ designates an eigenchannel of the scattering process. The angle $\pi \eta_{\Gamma}$ is the eigenphase shift in the eigenchannel $\Gamma$ owing to the residual interaction $V_{\text {res }}^{\text {ec }}$.

The asymptotic form of the standing-wave eigenfunction will be needed later when the incoming wave boundary conditions are applied. We obtain this form by using the standard asymptotic formula for the basis states in Eq. (9):

$$
\begin{aligned}
\left|\Phi^{N} \mu, \epsilon f, \sigma\right\rangle_{\mathcal{E}} & \underset{r \rightarrow \infty}{\rightarrow} \Theta_{\mu, \sigma}\left(\frac{2}{\pi k_{\mu}(\mathcal{E})}\right)^{1 / 2} \\
& \times \frac{1}{r} \sin \left[k_{\mu}(\mathcal{E}) r-\frac{1}{2} \pi l+\delta_{\mu}(\mathcal{E})\right] .
\end{aligned}
$$

Here $\Theta_{\mu, \sigma}$ comprises the wave function of the ionic core and the appropriate angular factors, $k_{\mu}(\mathcal{E})$ $=\left[2 m\left(\mathcal{E}-E_{\mu}\right)\right]^{1 / 2} / \hbar, l=3$, and

$$
\delta_{\mu}(\mathcal{E})=k_{\mu}^{-1}(\mathcal{E}) \ln \left[2 k_{\mu}(\mathcal{E}) r\right]+\arg \Gamma\left(l+1-i k_{\mu}^{-1}(\mathcal{E})\right) .
$$

Equation (12) is just the asymptotic formula for a particle in a Coulomb field, generalized, of course, to include the ionic-core states. The result of using Eqs. (11) and (12) in Eq. (9) is ${ }^{15}$

$$
\begin{aligned}
& \left|\Phi^{N} e \Gamma \mathcal{E}\right\rangle-\sum_{\mu, \sigma} \Theta_{\mu, \sigma}\left(\frac{2}{\pi k_{\mu}(\mathcal{E})}\right)^{1 / 2} \\
& \quad \times \frac{1}{r} \sin \left[k_{\mu}(\mathcal{E}) r-\frac{1}{2} \pi l+\delta_{\mu}(\mathcal{E})+\pi \eta_{\Gamma}\right] U_{\mu, \sigma ; \Gamma}(\mathcal{E}) \\
& \quad \text { as } r \rightarrow \infty,
\end{aligned}
$$

which shows explicitly that $\pi \eta_{\Gamma}$ is the eigenphase shift in the $\Gamma$ th channel due to $V_{\text {res }}^{\mathrm{ec}}$.

We have thus shown how to diagonalize the Hamiltonian submatrix in Eq。(6), which in the new representation becomes

$$
\left\langle\Phi^{N} e \Gamma^{\prime} \mathcal{E}^{\prime}|H| \Phi^{N} e \Gamma \mathcal{E}\right\rangle=\mathcal{E} \delta_{\Gamma^{\prime} \Gamma^{\prime}} \delta\left(\mathcal{E}^{\prime}-\mathcal{E}\right) .
$$

\section{RESONANCE ENERGY LEVELS}

Using the new representations for the discrete and continuum states obtained in Sec. II, the $\Lambda$ th standing-wave eigenfunction for the total Hamiltonian becomes [cf. Eq. (2)]

$$
\begin{aligned}
|\Lambda E\rangle \equiv \sum_{B}\left|\Phi^{N+1} \beta\right\rangle A_{\beta \Lambda}(E) & \\
& \quad+\sum_{\Gamma} \int d \mathcal{E}\left|\Phi^{N} e \Gamma \mathcal{E}\right\rangle B(\Gamma \mathcal{E} ; \Lambda E)
\end{aligned}
$$

Here the $A$ and $B$ coefficients satisfy the following linear equations [cf. Eqs. (3) and (4)]:

$$
\begin{aligned}
E_{\beta} A_{\beta \Lambda}(E)+\sum_{\Gamma^{\prime}} \int d \mathcal{E}^{\prime}\left\langle\Phi^{N+1} \beta\left|V_{\text {res }}\right|\right. & \left.\Phi^{N} e \Gamma^{\prime} \mathcal{E}^{\prime}\right\rangle B\left(\Gamma^{\prime} \mathcal{E}^{\prime} ; \Lambda E\right) \\
& =E A_{\beta \Lambda}(E), \\
\sum_{\beta^{\prime}}\left\langle\Phi^{N} e \Gamma \mathcal{E}\left|V_{\text {res }}\right| \Phi^{N+1} \beta^{\prime}\right\rangle A_{\beta^{\prime} \Lambda}(E) & +\mathcal{E} B(\Gamma \mathcal{E} ; \Lambda E) \\
= & E B(\Gamma \mathcal{E} ; \Lambda E)
\end{aligned}
$$

We thus see that in the new representation the discrete-continuum interaction stands out as the only one remaining; Fano's treatment of autoionization ${ }^{6}$ started from here. Our task in this section and Sec. IV is to obtain explicit expressions for the $A$ and $B$ coefficients, which amounts to obtaining the exact final-state eigenfunction $[\mathrm{Eq} .(15)]$ for our problem.

Solution of the linear equations above starts by 
using Eq. (17) to obtain the $B$ coefficients in terms of the $A$ coefficients and a set of integration constants $C$ :

$B(\Gamma \mathcal{E} ; \Lambda E)=\frac{\odot}{E-\mathcal{E}} \sum_{\beta^{\prime}}\left\langle\Phi^{N} e \Gamma \mathcal{E}\left|V_{\mathrm{res}}\right| \Phi^{N+1} \beta^{\prime}\right\rangle A_{\beta^{\prime} \Lambda}(E)$

$$
+\delta(E-\mathcal{E}) C_{\Gamma, \Lambda}(E),
$$

where $\beta$ indicates the Cauchy principal part is to be taken when integrating over the singularity.

Substituting Eq. (18) in Eq. (16) we obtain a linear relation between the $A$ and $C$ coefficients:

$$
\begin{aligned}
E_{\beta} A_{\beta \Lambda}(E)+\sum_{\beta^{\prime}}\left\{\sum_{\Gamma^{\prime}} \int d \mathcal{E}^{\prime}\left\langle\Phi^{N+1} \beta\left|V_{\text {res }}\right| \Phi^{N} e \Gamma^{\prime} \mathcal{E}^{\prime}\right\rangle \frac{\rho}{E-\mathcal{E}^{\prime}}\left\langle\Phi^{N} e \Gamma^{\prime} \mathcal{E}^{\prime}\left|V_{\text {res }}\right| \Phi^{N+1} \beta^{\prime}\right\rangle\right\} \\
\times A_{\beta^{\prime} \Lambda}(E)+\sum_{\Gamma^{\prime}}\left\langle\Phi^{N+1} \beta\left|V_{\text {res }}\right| \Phi^{N} e \Gamma^{\prime} E\right\rangle C_{\Gamma^{\prime}, \Lambda}(E)=E A_{\beta \Lambda}(E),(19)
\end{aligned}
$$

where we note that the matrix in curly brackets represents a second-order interaction between the discrete states due to their interaction with the continuum channels. In particular, this interaction shifts the energies of the discrete levels. ${ }^{16}$ Following Fano $^{6}$ we define

$$
\begin{aligned}
F_{\beta \beta^{\prime}}(E) \equiv & \sum_{\Gamma^{\prime}} \int d \mathcal{E}^{\prime}\left\langle\Phi^{N+1} \beta\left|V_{\text {res }}\right| \Phi^{N} e \Gamma^{\prime} \mathcal{E}^{\prime}\right\rangle \\
& \times \frac{\odot}{E-\mathcal{E}^{\prime}}\left\langle\Phi^{N} e \Gamma^{\prime} \mathcal{E}^{\prime}\left|V_{\text {res }}\right| \Phi^{N+1} \beta^{\prime}\right\rangle .
\end{aligned}
$$

We wish now to diagonalize the matrix $E_{\beta} \delta_{\beta \beta^{\prime}}$ $+F_{\beta \beta^{\prime}}(E)$ according to the equation

$$
E_{\beta} A_{\beta \Lambda}(E)+\sum_{\beta^{\prime}} F_{\beta \beta^{\prime}}(E) A_{\beta^{\prime} \Lambda}(E)=E_{\gamma}(E) A_{\beta \Lambda}(E),
$$

where the $E_{\gamma}(E)$ are the eigenvalues and where we have explicitly noted that these eigenvalues vary with the total energy $E$. We shall denote the eigenstates of Eq. (21) by $\left|\Phi^{N+1} \gamma\right\rangle$.

This calculation has two important results. One is that in the new representation for the discrete states, Eq. (19) gives an explicit solution for the $A$ coefficients in terms of the $C$ 's, namely,

$$
A_{\gamma \Lambda}(E)=\frac{1}{E-E_{\gamma}(E)} \sum_{\Gamma^{\prime}}\left\langle\Phi^{N+1} \gamma\left|V_{\text {res }}\right| \Phi^{N} e \Gamma^{\prime} E\right\rangle C_{\Gamma^{\prime}, \Lambda}(E) .
$$

The other is that the resonance behavior of the exact final-state wave function will be seen later on to arise from the energy denominator in Eq. (22). In particular, the energy $E$ at which the denominator vanishes, i.e.,

$$
E=E_{\gamma}(E) \text {, }
$$

approximates the energy of the $\gamma$ th peak in the observed photoabsorption spectrum. Note that since $E_{\gamma}(E)$ depends on $E$, Eq. (23) must be solved iteratively or else graphically.

We have thus shown explicitly how to obtain expressions for $A_{\gamma \Lambda}(E)$ and $B(\Gamma \mathcal{E} ; \Lambda E)$ in terms of new coefficients $C_{\Gamma, \Lambda}(E)$ for energies $E$ in the autoionization region. In Sec. IV we shall show that the $C_{\Gamma, \Lambda}(E)$ coefficients diagonalize yet another reaction matrix which takes into account the interaction between the continuum channels due to their mutual interaction with the discrete levels.

In the rest of this section we consider those discrete levels of the configuration $\Phi^{N+1}$ which lie below the onset of some or all continuum channels. (Note that we are considering the Rydberg series preceding each continuum threshold as merely an appendage of the continuum, as discussed in Sec. II. ) Those discrete levels below all continuum thresholds do not autoionize, but their energy position is nevertheless shifted through interaction with the continuum channels. In short, we wish to obtain here the total final-state wave function $|\Lambda E\rangle$ for discrete energies $E=E_{6}$. This wave function has still the form (15), but we must solve for the $A$ and $B$ coefficients in a slightly different way. In the expression for the $B$ coefficients [Eq. (18)] the second term does not now appear since $E_{6}<\mathcal{E}$. Hence we have

$$
B\left(\Gamma \mathcal{E} ; \Lambda E_{6}\right)=\frac{1}{E_{\delta}-\mathcal{E}} \sum_{\beta^{\prime}}\left\langle\Phi^{N} e \Gamma \mathcal{E}\left|V_{\text {res }}\right| \Phi^{N+1} \beta^{\prime}\right\rangle A_{\beta^{\prime} \Lambda}\left(E_{\delta}\right) \text {. }
$$

Substituting Eq. (18') in Eq. (16) we obtain an eigenvalue equation for the $A$ coefficients (we henceforth drop the symbol $\Lambda$ since it is superfluous):

$$
E_{\beta} A_{\beta}\left(E_{6}\right)+\sum_{\beta^{\prime}} F_{\beta \beta^{\prime}}\left(E_{6}\right) A_{\beta^{\prime}}\left(E_{6}\right)=E_{6} A_{\beta}\left(E_{6}\right) .
$$

Note that Eq. (21') differs from Eq. (21) in that here $F_{\beta \beta^{\prime}}\left(E_{6}\right)$ depends on the eigenvalue $E_{6}$, and hence Eq. (21') must be solved iteratively, unlike Eq. (21). [That is, we must diagonalize the matrix $E_{\beta} \delta_{\beta \beta^{\prime}}+F_{\beta \beta^{\prime}}\left(E_{\delta}\right)$, as before, but now at the same time we must require that one of the eigenvalues equal $E_{6}$.]

The energies $E_{0}$ which satisfy Eq. $\left(21^{\prime}\right)$ are the experimentally observable energies for absorption to those levels of the configuration $\Phi^{N+1}$ which lie below the continuum channel onsets. The $A_{\beta}\left(E_{6}\right)$ coefficients which solve Eq. $\left(21^{\prime}\right)$ are determined up to normalization and may be used with Eq. (18') to define the final-state eigenfunction in Eq. (15); this eigenfunction will be used in later sections to compute oscillator strengths. The final-state eigenfunction $\left|E_{6}\right\rangle$ must have discrete normalization, which implies that the $A$ coefficients are normalized according to the relation 


$$
\left\langle E_{6} \mid E_{\delta}\right\rangle=1=\sum_{\beta^{\prime} \beta} A_{\beta^{\prime}}{ }^{\dagger}\left(E_{\delta}\right) \quad\left\{\delta_{\beta^{\prime} \beta}+\sum_{\Gamma} \int d \mathcal{E}\left\langle\Phi^{N+1} \beta^{\prime}\left|V_{\text {res }}\right| \Phi^{N} e \Gamma \mathscr{E}\right\rangle \frac{1}{\left(E_{\delta}-\mathcal{E}\right)^{2}}\left\langle\Phi^{N} e \Gamma \mathscr{E}\left|V_{\text {res }}\right| \Phi^{N+1} \beta\right\rangle\right\} A_{\beta}\left(E_{\delta}\right),
$$

where

$$
\left|E_{\delta}\right\rangle=\sum_{\beta}\left\{\left|\Phi^{N+1} \beta\right\rangle+\sum_{\Gamma} \int d \mathcal{E}\left|\Phi^{N} e \Gamma \mathcal{E}\right\rangle \frac{1}{E_{\delta}-\mathcal{E}}\left\langle\Phi^{N} e \Gamma \mathcal{E}\left|V_{\text {res }}\right| \Phi^{N+1} \beta\right\rangle\right\} A_{\beta}\left(E_{\delta}\right) .
$$

Finally, when the total energy $E$ is above some continuum thresholds but below others, the $B(\Gamma \mathcal{E} ; \Lambda E)$ coefficients are obtained either from Eq. (18), if $\Gamma$ is an open channel, or from Eq. $\left(18^{\prime}\right)$, if $\Gamma$ is a closed channel. Substitution of these equations in Eq. (16) gives a result identical to Eq. (19), except that the terms

$$
\left\langle\Phi^{N+1} \beta\left|V_{\text {res }}\right| \Phi^{N} e \Gamma^{\prime} E\right\rangle C_{\Gamma^{\prime}, \Lambda}(E)
$$

are summed only over those $\Gamma^{\prime}$ channels which are open. Otherwise, just as for the case of all chan- nels open, the resonance energies are obtained from Eqs. (20)-(23). The $C_{\Gamma, \Lambda}(E)$ coefficients are determined in Sec. IV.

\section{DETERMINATION OF FINAL-STATE EIGENVECTOR}

In Sec. III we determined the final-state eigenfunction for discrete energies. For energies in the autoionization region (i.e., above the lowest continuum channel onset) we obtained the final-state eigenfunction in terms of the yet to be defined coefficients $C_{\Gamma, \Lambda}(E)$, i. e.,

$$
\begin{aligned}
|\Lambda E\rangle= & \sum_{\Gamma}^{\text {open }}\left\{\left|\Phi^{N} e \Gamma E\right\rangle+\sum_{\gamma}\left|\Phi^{N+1} \gamma\right\rangle \frac{1}{E-E_{\gamma}(E)}\left\langle\Phi^{N+1} \gamma\left|V_{\text {res }}\right| \Phi^{N} e \Gamma E\right\rangle\right. \\
& \left.+\sum_{\gamma} \sum_{\Gamma^{\prime}} \int d \mathcal{E}\left|\Phi^{N} e \Gamma^{\prime} \mathcal{E}\right\rangle \frac{p}{E-\mathcal{E}}\left\langle\Phi^{N} e \Gamma^{\prime} \mathcal{E}\left|V_{\text {res }}\right| \Phi^{N+1} \gamma\right\rangle \frac{1}{E-E_{\gamma}(E)}\left\langle\Phi^{N+1} \gamma\left|V_{\text {res }}\right| \Phi^{N} e \Gamma E\right\rangle\right\} C_{\Gamma, \Lambda}(E) .
\end{aligned}
$$

Here we have substituted Eqs. (22) and (18) [or Eq. $\left(18^{\prime}\right)$ for the $\Gamma^{\prime}$ channels which are closed] in Eq. (15) and transformed to the new representation for the discrete states. Note that the sum over $\Gamma$ is only over those channels which are open at energy $E$, whereas the sum over $\Gamma^{\prime}$ is over all continuum channels.

To obtain the coefficients $C_{\Gamma, \Lambda}(E)$ we note initially that Eq. (26) has the following form in abstract operator notation:

$|\Lambda E\rangle=\sum_{\Gamma}^{\text {open }}\left\{\left|\Phi^{N} e \Gamma E\right\rangle+\frac{1}{E-H^{\prime}} \bar{K}(E)\left|\Phi^{N} e \Gamma E\right\rangle\right\} C_{\Gamma, \Lambda}(E)$.

Here $H^{\prime}$ assumes the value $E_{\gamma}(E)$ for discrete states $\left|\Phi^{N+1} \gamma\right\rangle$ and the value $\mathcal{E}$ for continuum states $\left|\Phi^{N} e \Gamma \mathcal{E}\right\rangle . \bar{K}(E)$ is another reaction matrix, which will be shown later to satisfy the usual integral equation [cf. Eq. (10)]

$$
\bar{K}(E)=V^{\prime}+V^{\prime} \odot /\left(E-H^{\prime}\right)^{-1} \bar{K}(E),
$$

where, of course, $V^{\prime} \equiv H-H^{\prime}$. Formal analogy of Eq. (27) with Eq. (9) identifies the $C_{\Gamma, \Lambda}(E)$ as having the form

$$
C_{\Gamma, \Lambda}(E)=\bar{U}_{\Gamma, \Lambda}(E) \cos \pi \varphi_{\Lambda},
$$

where $\bar{U}_{\Gamma, \Lambda}(E)$ is an orthogonal matrix and $\pi \varphi_{\Lambda}$ is a phase shift. These are determined from the eigenvalue equation analogous to Eq. (11), i. e.,

$$
\sum_{\Gamma^{\prime}}\{\bar{K}(E)\}_{\Gamma, \Gamma^{\prime}}, \bar{U}_{\Gamma^{\prime}, \Lambda}(E)=-\pi^{-1}\left(\tan \pi \varphi_{\Lambda}\right) \bar{U}_{\Gamma, \Lambda}(E),
$$

where the matrix $\{\bar{K}(E)\}_{\Gamma, \Gamma}$, is seen by comparison of Eqs. (26) and (27) to be given by

$$
\begin{aligned}
\{\bar{K}(E)\}_{\Gamma, \Gamma^{\prime}}= & \sum_{\gamma}\left\langle\Phi^{N} e \Gamma E\left|V_{\text {res }}\right| \Phi^{N+1} \gamma\right\rangle \\
& \times \frac{1}{E-E_{\gamma}(E)}\left\langle\Phi^{N+1} \gamma\left|V_{\text {res }}\right| \Phi^{N} e \Gamma^{\prime} E\right\rangle .
\end{aligned}
$$

Note that Eq. (31) gives $\{\bar{K}(E)\}_{\Gamma, \Gamma}$, explicitly. We do not have to solve an integral equation as was done in Sec. II. This results from the representation we use in solving Eq. (28), as shown in more detail in the rest of this section. Beforehand, we note that Eqs. (29) $-(31)$ determine the $C_{\Gamma, \Lambda}(E)$ coefficients, so that now the final-state standing-wave eigenvector [Eq. (26)] is completely defined. Also note that we may write Eq. (26) in a form which emphasizes its resonance structure. That is, following Fano ${ }^{6}$ we define an "augmented" discrete state, denoted by $\left|\Phi^{N+1} \gamma+\right\rangle$, which includes a contribution from the continuum states, i.e.,

$$
\begin{aligned}
\left|\Phi^{N+1} \gamma+\right\rangle \equiv & \left|\Phi^{N+1} \gamma\right\rangle+\sum_{\Gamma} \int d \mathcal{E}\left|\Phi^{N} e \Gamma \mathcal{E}\right\rangle \\
& \times \frac{\odot}{E-\mathcal{E}}\left\langle\Phi^{N} e \Gamma \mathcal{E}\left|V_{\text {res }}\right| \Phi^{N+1} \gamma\right\rangle .
\end{aligned}
$$

Using this augmented discrete state and the expression for the $C_{\Gamma, \Lambda}(E)$ coefficients $[\mathrm{Eq} .(29)]$, the $\Lambda$ th standing-wave eigenfunction [Eq. (26)] assumes the following form: 


$$
|\Lambda E\rangle=\sum_{\Gamma}^{\text {open }}\left\{\left|\Phi^{N} e \Gamma E\right\rangle+\sum_{\gamma}\left|\Phi^{N+1} \gamma+\right\rangle \frac{1}{E-E_{\gamma}(E)}\left\langle\Phi^{N+1} \gamma\left|V_{\text {res }}\right| \Phi^{N} e \Gamma E\right\rangle\right\} \bar{U}_{\Gamma, \Lambda}(E) \cos \pi \varphi_{\Lambda}
$$

in which the resonance denominator $\left[E-E_{\gamma}(E)\right]$ is prominent. We shall use this form of the finalstate eigenfunction in Sec. V to obtain partial and total cross sections for photoabsorption.

In order to see how $H^{\prime}$ is defined, let us review the properties of the discrete and continuum representations we obtained in Secs. II and III. These are [cf. Eqs. (5), (14), and (21)]

$$
\begin{aligned}
& \left\langle\Phi^{N+1} \gamma^{\prime}|H+F(E)| \Phi^{N+1} \gamma\right\rangle=E_{\gamma}(E) \delta_{\gamma^{\prime} \gamma}, \\
& \left\langle\Phi^{N} e \Gamma^{\prime} \mathcal{E}^{\prime}|H| \Phi^{N} e \Gamma \mathcal{E}\right\rangle=\mathcal{E} \delta_{\Gamma^{\prime} \Gamma} \delta\left(\mathcal{E}^{\prime}-\mathcal{E}\right), \\
& \left\langle\Phi^{N+1} \gamma|H| \Phi^{N} e \Gamma \mathcal{E}\right\rangle=\left\langle\Phi^{N+1} \gamma\left|V_{\text {res }}\right| \Phi^{N} e \Gamma \mathcal{E}\right\rangle,
\end{aligned}
$$

where $V_{\text {res }} \equiv H-H_{\text {mod }}$. The properties (34a)-(34c) may be described most simply if we use projection operators to distinguish our final basis states. We define the operators $P_{D}$ and $P_{C}$ (for the discrete and continuum states, respectively) as follows:

$$
\begin{aligned}
& P_{D} \equiv \sum_{\gamma}\left|\Phi^{N+1} \gamma\right\rangle\left\langle\Phi^{N+1} \gamma\right|, \\
& P_{C} \equiv \sum_{\Gamma} \int d \mathcal{E}\left|\Phi^{N} e \Gamma \mathcal{E}\right\rangle\left\langle\Phi^{N} e \Gamma \mathcal{E}\right| .
\end{aligned}
$$

Since these two operators span our space in the approximation that we only consider the configurations $\Phi^{N+1}$ and $\Phi^{N} e$, they have the closure property $P_{D}+P_{C}=1$. They also have the following standard relations:

$$
\begin{array}{r}
\left.P_{D}\left|\Phi^{N+1} \gamma\right\rangle=\left|\Phi^{N+1} \gamma\right\rangle \text { (alternatively } P_{D} P_{D}=P_{D}\right) \\
P_{C}\left|\Phi^{N} e \Gamma E\right\rangle=\left|\Phi^{N} e \Gamma E\right\rangle\left(\text { al ternatively } P_{C} P_{C}=P_{C}\right) \\
P_{D}\left|\Phi^{N} e \Gamma E\right\rangle=P_{C}\left|\Phi^{N+1} \gamma\right\rangle=0 \\
\text { (alternatively } \left.P_{D} P_{C}=P_{C} P_{D}=0\right)
\end{array}
$$

Using these projection operators we see that the properties (34a) and (34b) imply that the submatrices $P_{D}\{H+F(E)\} P_{D}$ and $P_{C} H P_{C}$ are diagonal in our representation of states. Therefore, in partitioning the Hamiltonian such that $H=H^{\prime}+V^{\prime}$, we choose

$$
H^{\prime}=P_{D}\{H+F(E)\} P_{D}^{-}+P_{C} H P_{C} .
$$

Since $V^{\prime} \equiv H-H^{\prime}$ we obtain

$$
V^{\prime}=P_{C} V_{\text {res }} P_{D}+P_{D} V_{\text {res }} P_{C}-P_{D} F(E) P_{D}
$$

where we have used Eq. (34c), which may be expressed as $P_{D} H P_{C}=P_{D} V_{\text {res }} P_{C}$.

Having defined $H^{\prime}$ and $V^{\prime}$ we may obtain $\bar{K}(E)$ by taking projections of Eq. (28) and using Eqs. (36) and (37). This straightforward calculation gives the following explicit relations for $\bar{K}(E)$ :

$$
\begin{aligned}
& P_{D} \bar{K}(E) P_{C}=P_{D} V_{\text {res }} P_{C}, \\
& P_{C} \bar{K}(E) P_{C}=P_{C} V_{\text {res }} P_{D} \frac{1}{E-H^{\prime}} P_{D} V_{\text {res }} P_{C}, \\
& P_{D} \bar{K}(E) P_{D}=0, \\
& P_{C} \bar{K}(E) P_{D}=P_{C} V_{\text {res }} P_{D} .
\end{aligned}
$$

Note that the matrix $\{\bar{K}(E)\}$, given by Eq. (31), is a submatrix of (38b). Also, Eqs. (38a) and (38b) enable us to make direct comparison between Eqs.

(26) and (27).

\section{BOUNDARY CONDITIONS AND CROSS SECTIONS}

In Sec. IV we determined a complete set of standing-wave eigenfunctions for the eigenchannels $\Lambda$. These eigenfunctions do not satisfy the boundary condition for a photoionization process, namely, that there is an outgoing electron in a particular observable channel, characterized by the state of the ionic core and its coupling with the outgoing electron. [Recall that the observable states of the ionic core plus outgoing electron were obtained from the diagonalization leading to the states $\left|\Phi^{N} \mu, \epsilon f, \sigma\right\rangle$ in Eq. (7). ] We must obtain the desired final-state eigenfunction as a linear combination of our complete set of standing-wave states, i. e.,

$$
\left|\psi_{E}\right\rangle^{\left(\mu, \sigma_{-}\right)}=\sum_{\Lambda}^{\text {open }}|\Lambda E\rangle \chi_{\Lambda}{ }^{\left(\mu, \sigma_{-}\right)} .
$$

Here $\left|\psi_{E}\right\rangle^{\left(\mu, \sigma_{-}\right)}$denotes the desired incoming-wave solution for the $(\mu, \sigma)$ state of the ionic core plus outgoing electron. The set of coefficients $\chi_{\Lambda}^{(\mu, \sigma-)}$ are determined by application of the so-called "incoming-wave boundary condition" for the channel $(\mu, \sigma)$, which requires that asymptotically the amplitudes for all outgoing waves in channels other than $(\mu, \sigma)$ vanish.

The asymptotic form of $\left|\psi_{E}\right\rangle^{(\mu, \sigma-)}$ is obtained by using the known asymptotic form of the continuum basis states $\left|\Phi^{N} e \Gamma E\right\rangle$ [given by Eq. (13)] in Eq. (26) for the state $|\Lambda E\rangle$. We thus ${ }^{15}$ obtain

$$
\left|\psi_{E}\right\rangle^{(\mu, \sigma-)} \rightarrow \sum_{\Lambda}^{\text {open open }} \sum_{\Gamma} \sum_{\mu, \sigma} \Theta_{\mu, \sigma}\left(\frac{2}{\pi k_{\mu}(E)}\right)^{1 / 2} \frac{1}{r} \sin \left[k_{\mu}(E) r-\frac{1}{2} \pi l+\delta_{\mu}(E)+\pi \eta_{\Gamma}+\pi \varphi_{\Lambda}\right] U_{\mu, \sigma ; \Gamma}(E) \bar{U}_{\Gamma, \Lambda}(E) \chi_{\Lambda}^{(\mu, \sigma-)} \text { as } r \rightarrow \infty,
$$

where we have used Eqs. (29) and (30) and the definitions of Sec. II. We see explicitly that the interaction between continuum channels due to their mu- tual coupling with the discrete states produces the phase shift $\pi \varphi_{\Lambda}$. To apply the incoming-wave boundary condition we use the identity $\sin x$ 
$=\left(e^{i x}-e^{i x}\right) / 2 i$ and require that the coefficients of the outgoing waves (i.e., $e^{i x}$ ) vanish for all channels other than $(\mu, \sigma)$. We thus obtain the following equations:

$$
\begin{aligned}
\sum_{\Gamma}^{\text {open open }} \sum_{\Lambda}^{i \pi\left(\eta_{\Gamma}+\varphi_{\Lambda}\right)} U_{\mu^{\prime}, \sigma^{\prime} ; \Gamma}(E) \chi_{\Lambda}^{(\mu, \sigma-)} & \\
& =\delta_{\mu^{\prime} \mu} \delta_{\sigma^{\prime} \sigma},
\end{aligned}
$$

$$
\chi_{\Lambda}{ }^{(\mu, \sigma-)}=\sum_{\Gamma}^{\text {open }} e^{-i \pi \varphi} \Lambda \tilde{\bar{U}}_{\Lambda, \Gamma}(E) e^{-i \pi \eta} \Gamma \tilde{U}_{\Gamma ; \mu, \sigma}(E)
$$

Having thus obtained the exact incoming-wave eigenfunctions of the total Hamiltonian we are ready to compute cross sections.

The dipole amplitude for transition from an initial state, which we denote by $\left\langle\psi_{0}\right|$, to the observable channel $(\mu, \sigma)$ is given by

$$
\begin{array}{r}
\left\langle\psi_{0}\left|\sum_{i} \overrightarrow{\mathrm{r}}_{i}\right| \psi_{E}\right\rangle^{(\mu, \sigma-)}=\sum_{\Lambda}^{\text {open open }} \sum_{\Gamma}\left\{\left\langle\psi_{0}\left|\sum_{i} \overrightarrow{\mathrm{r}}_{i}\right| \Phi^{N} e \Gamma E\right\rangle+\sum_{\gamma}\left\langle\psi_{0}\left|\sum_{i} \overrightarrow{\mathrm{r}}_{i}\right| \Phi^{N+1} \gamma+\right\rangle \frac{1}{E-E_{\gamma}(E)}\left\langle\Phi^{N+1} \gamma\left|V_{\mathrm{res}}\right| \Phi^{N} e \Gamma E\right\rangle\right\} \\
\times \bar{U}_{\Gamma, \Lambda}(E)\left(\cos \pi \varphi_{\Lambda}\right) \chi_{\Lambda}^{\left(\mu, \sigma^{-}\right)} .
\end{array}
$$

Note that the dipole matrix element $\left\langle\psi_{0}\left|\Gamma_{,} \overrightarrow{\mathrm{r}}_{i}\right| \Phi^{N+1} \gamma+\right\rangle$ is probably only slightly affected by the continuum contribution implied by "+" [cf. Eq. (32)]. This is because, as discussed in the Introduction, in the rare earths the dipole matrix element for the transition $4 d \rightarrow 4 f$ is very much greater than for the transitions $4 d \rightarrow \epsilon f$. Furthermore, we clearly see that the tran- sition matrix element has resonances at $E=E_{\gamma}(E)$. [Note also that we have used the length formula for the transition operators, as required when solving for the exact eigenstates of a nonlocal Hamiltonian. ${ }^{17}$ ]

For completeness, we give the dipole amplitude for transition to a level of the configuration $\Phi^{N+1}$ lying below the onset of any continuum channel. That is,

$$
\left\langle\psi_{0}\left|\sum_{i} \overrightarrow{\mathbf{r}}_{i}\right| E_{\delta}\right\rangle=\sum_{\beta}\left\{\left\{\psi_{0}\left|\sum_{i} \overrightarrow{\mathrm{r}}_{i}\right| \Phi^{N+1} \beta\right\rangle+\sum_{\Gamma} \int d \mathcal{E}\left\langle\psi_{0}\left|\sum_{i} \overrightarrow{\mathbf{r}}_{i}\right| \Phi^{N} e \Gamma \mathcal{E}\right\rangle \frac{1}{E_{\delta}-\mathcal{E}}\left\langle\Phi^{N} e \Gamma \mathcal{E}\left|V_{\mathrm{res}}\right| \Phi^{N+1} \beta\right\rangle\right\} A_{\beta}\left(E_{6}\right),
$$

where $\left|E_{6}\right\rangle$ is obtained from Eq. (25) and the $A_{\beta}\left(E_{6}\right)$ are obtained from Eqs. $\left(21^{\prime}\right)$ and (24). We see that the $\left|\Phi^{N+1} \beta\right\rangle$ states have a small continuum contribution and are superposed with coefficients $A_{\beta}\left(E_{6}\right)$. The oscillator strength for this transition is given by

$$
f_{6}=\frac{2}{3}\left(E_{6}-E_{0}\right)\left|\left\langle\psi_{0}\left|\sum_{i} \overrightarrow{\mathrm{r}}_{i}\right| E_{6}\right\rangle\right|^{2}
$$

where $E_{0}$ is the energy of the ground state $\left\langle\psi_{0}\right\rangle$.

Returning to the autoionization region, the partial cross section for transition to the channel $(\mu, \sigma)$ is given by

$$
\sigma_{E}^{(\mu, \sigma-)}=\frac{4}{3} \frac{\pi^{2} e^{2} \hbar}{m c}\left(E-E_{0}\right)\left|\left\langle\psi_{0}\left|\sum_{i} \overrightarrow{\mathrm{r}}_{i}\right| \psi_{E}\right\rangle^{(\mu, \sigma-)}\right|^{2},
$$

where the dipole matrix element is obtained from
Eq. (43). The total cross section for transition to all open channels at energy $E$ is given by

$$
\sigma_{E}^{\text {TOT }}=\frac{4}{3} \frac{\pi^{2} e^{2} \hbar}{m c}\left(E-E_{0}\right) \sum_{\Lambda}^{\text {open }}\left|\left\langle\psi_{0}\left|\sum_{i} \overrightarrow{\mathrm{r}}_{i}\right| \Lambda E\right\rangle\right|^{2},
$$

where we have made use of the relation

$$
\sum_{\mu, \sigma} \chi_{\Lambda}^{\left(\mu, \sigma_{-}\right)} \chi_{\Lambda^{\prime}}{ }^{\dagger\left(\mu, \sigma_{-}\right)}=\delta_{\Lambda, \Lambda^{\prime}} .
$$

For simplicity we now identify the eigenvectors of the matrix $\{\bar{K}(E)\}$ by [cf. Eq. (30)]

$$
\left|\bar{\Psi}_{\Lambda}(E)\right\rangle \equiv \sum_{\Gamma}\left|\Phi^{N} e \Gamma E\right\rangle \bar{U}_{\Gamma, \Lambda}(E) .
$$

Using these new continuum states and Eq. (33) for the standing-wave eigenvector for $|\Lambda E\rangle$, Eq. (47) may be written as

$$
\sigma_{E}^{\mathrm{TOT}}=\frac{4}{3} \frac{\pi^{2} e^{2} \hbar}{m c}\left(E-E_{0}\right) \sum_{\Lambda}^{\text {open }}\left|\left\langle\psi_{0}\left|\sum_{i} \overrightarrow{\mathrm{r}}_{i}\right| \bar{\Psi}_{\Lambda}(E)\right\rangle+\sum_{\gamma}\left\langle\psi_{0}\left|\sum_{i} \overrightarrow{\mathrm{r}}_{i}\right| \Phi^{N+1} \gamma+\right\rangle \frac{1}{E-E_{\gamma}(E)}\left\langle\Phi^{N+1} \gamma\left|V_{\mathrm{res}}\right| \bar{\Psi}_{\Lambda}(E)\right\rangle\right|^{2} \cos ^{2} \pi \varphi_{\Lambda} .
$$

Using Eq. (31), we write Eq. (30) in the new continuum representation as

$$
\begin{array}{r}
\sum_{\gamma}\left\langle\bar{\Psi}_{\Lambda}(E)\left|V_{\text {res }}\right| \Phi^{N+1} \gamma\right\rangle \frac{1}{E-E_{\gamma}(E)}\left\langle\Phi^{N+1} \gamma\left|V_{\text {res }}\right| \Psi_{\Lambda}(E)\right\rangle \\
=-\pi^{-1} \tan \pi \varphi_{\Lambda} .
\end{array}
$$

Following Fano ${ }^{6}$ we define

$$
\begin{aligned}
\left\langle\bar{\Psi}_{\Lambda}(E)\left|V_{\text {res }}\right| \Phi^{N+1} \gamma\right\rangle \frac{1}{E-E_{\gamma}(E)} & \left\langle\Phi^{N+1} \gamma\left|V_{\text {res }}\right| \bar{\Psi}_{\Lambda}(E)\right\rangle \\
& \equiv-\pi^{-1} \tan \pi \varphi_{\Lambda}^{\gamma},
\end{aligned}
$$


where $\varphi_{\Lambda}^{\gamma}$ is the phase shift that would be obtained if there were only a single discrete resonance. Multiplying and dividing the second term inside the modulus in Eq. (49) by $\left\langle\bar{\Psi}_{\Lambda}(E)\left|V_{\text {res }}\right| \Phi^{N+1} \gamma\right\rangle$ we obtain the following parametrized form for the total cross section:

$$
\sigma_{E}^{\mathrm{TOT}}=\frac{4}{3} \frac{\pi^{2} e^{2} \hbar}{m c}\left(E-E_{0}\right) \sum_{\Lambda}^{\text {open }}\left\langle\psi_{0}\left|\sum_{i} \overrightarrow{\mathrm{r}}_{i}\right| \bar{\Psi}_{\Lambda}(E)\right\rangle^{2} \cos ^{2} \pi \varphi_{\Lambda}\left|1-\sum_{\gamma} q_{\Lambda}^{\gamma} \tan \pi \varphi_{\Lambda}^{\gamma}\right|^{2}
$$

where we have defined

$$
q_{\Lambda}^{\gamma} \equiv \frac{\left\langle\psi_{0}\left|\sum_{i} \overrightarrow{\mathrm{r}}_{i}\right| \Phi^{N+1} \gamma+\right\rangle}{\pi\left\langle\psi_{0}\left|\sum_{i} \overrightarrow{\mathrm{r}}_{i}\right| \bar{\Psi}_{\Lambda}(E)\right\rangle\left\langle\bar{\Psi}_{\Lambda}(E)\left|V_{\mathrm{res}}\right| \Phi^{N+1} \gamma\right\rangle} .
$$

Note that Eq. (52) represents an incoherent sum over the partial cross sections for transition to the states $\left|\Psi_{\Lambda}(E)\right\rangle$ and that each partial cross section has exactly the form of Fano's parametrized crosssection formula for the case of many discrete states interacting with a single continuum channel. ${ }^{18}$ Since Eq. (52) involves an incoherent sum over the $\Lambda$ states, the total cross section does not go to zero each time a resonance is crossed (i.e., each time the $\Lambda$ th partial cross section has a zero as $\tan \pi \varphi_{\Lambda}^{\gamma}$ goes from $-\infty$ to $+\infty$ ). Note further that $q_{\Lambda}^{\gamma}$ is large since the dipole transitions $4 d \rightarrow 4 f$ are much stronger than $4 d \rightarrow \epsilon f$.

One may also easily see from Eq. (52) that the total cross section does not diverge when $\tan \pi \varphi_{\Lambda}^{\gamma}$ does diverge. This is because $\cos ^{2} \pi \varphi_{\Lambda}$ $=\left(1+\tan ^{2} \pi \varphi_{\Lambda}\right)^{-1}=\left[1+\left(\sum, \gamma \tan \pi \varphi_{\Lambda}^{\gamma}\right)^{2}\right]^{-1}$ and hence, the total cross section near a resonance is given by

$$
\begin{aligned}
\sigma_{E \approx E_{\gamma}}^{\text {TOT }}(E) \approx & \frac{4}{3} \frac{\pi^{2} e^{2} \hbar}{m c}\left[E_{\gamma}(E)-E_{0}\right] \\
& \times \sum_{\Lambda}^{\text {open }}\left\langle\psi_{0}\left|\sum_{i} \overrightarrow{\mathrm{r}}_{i}\right| \bar{\Psi}_{\Lambda}(E)\right\rangle^{2}\left(q_{\Lambda}^{\gamma}\right)^{2} .
\end{aligned}
$$

Substituting Eq. (53) for $q_{\Lambda}^{\gamma}$ in Eq. (54), we find that the cross section at a resonance has the magnitude

$$
\begin{aligned}
\sigma_{E=E_{\gamma}}^{\operatorname{ToT}}(E)= & \frac{4}{3} \frac{\pi^{2} e^{2} \hbar}{m c}\left[E_{\gamma}(E)-E_{0}\right] \\
& \times \sum_{\Lambda}^{\text {open }}\left|\frac{\left\langle\psi_{0} ! \sum_{i} \overrightarrow{\mathrm{r}}_{i} \mid \Phi^{N+1} \gamma+\right\rangle}{\pi\left\langle\bar{\Psi}_{\Lambda}(E) ! V_{\text {res }} \mid \Phi^{N+1} \gamma\right\rangle}\right|^{2} .
\end{aligned}
$$

Note that if we define a partial width for decay of the resonance $\gamma$ to the continuum channel $\Lambda$ as

$$
\Gamma_{\gamma \Lambda} \equiv 2 \pi\left\langle\Phi^{N+1} \gamma\left|V_{\text {res }}\right| \bar{\Psi}_{\Lambda}(E)\right\rangle^{2},
$$

and define the total width for the decay of the resonance $\gamma$ as $\Gamma_{\gamma} \equiv \sum_{\Lambda} \Gamma_{\gamma \Lambda}$, then we see that the magnitude of the cross section at the resonance $\gamma$ is inversely proportional to its total width, i.e.,

$\sigma_{E=E_{\gamma}}^{\mathrm{TOT}}(E)=\frac{4}{3} \frac{\pi^{2} e^{2} \hbar}{m c}\left[E_{\gamma}(E)-E_{0}\right] \frac{\left\langle\psi_{0}\left|\sum_{i} \vec{r}_{i}\right| \Phi^{N+1} \gamma+\right\rangle^{2}}{\frac{1}{2} \pi \Gamma_{\gamma}}$.

Note that the calculated resonance peaks do not, in general, occur at the resonance energy. This may be seen for an isolated resonance in Fig. 1 of
Ref. 6. However, since our $q$ values are fairly large, we do not expect the calculated peaks to be too far from the calculated resonance energies.

\section{SUMMARY AND PRESCRIPTION FOR NUMERICAL CALCULATIONS}

In this section we outline the step-by-step procedure necessary to calculate photoabsorption cross sections for the rare earths and, probably, for the transition metals and other elements for which the effect of the potential barrier is crucial. We assume a complete set of independent-particle-model wave functions. From these, linear combinations of Slater determinants are to be formed in some coupling scheme (usually $L-S$ coupling) for the configurations $\Phi^{N+1}$ and $\Phi^{N} \in f$. The resultant states are denoted by $\left|\Phi^{N+1} \alpha\right\rangle$ and $\left|\Phi^{N} \lambda, \epsilon f, \rho\right\rangle$, respectively, where the Greek letters identify both the states and the coupling scheme. Our prescription is as follows:

(i) Diagonalize $\left\langle\Phi^{N+1} \alpha^{\prime}|H| \Phi^{N+1} \alpha\right\rangle$, as in Eq. (5). Denote the eigenvectors by $\left|\Phi^{N+1} \beta\right\rangle$ and their energies by $E_{\beta}$. Use the methods of theoretical spectroscopy discussed in detail by Sugar. ${ }^{5}$

(ii) Diagonalize $\left\langle\Phi^{N} \lambda^{\prime}, \epsilon^{\prime} f, \rho^{\prime}|H| \Phi^{N} \lambda, \epsilon f, \rho\right\rangle$. This is accomplished in two steps. First, diagonalize

$$
\left\langle\Phi^{N} \lambda^{\prime}, \epsilon^{\prime} f, \rho^{\prime}\left|H_{\text {mod }}+V_{\text {res }}^{\text {core }}\right| \Phi^{N} \lambda, \epsilon f, \rho\right\rangle
$$

and denote the eigenvectors by $\left|\Phi^{N} \mu, \epsilon f, \sigma\right\rangle$, as indicated in Eqs. (6) and (7). Second, solve Eq. (10) for the matrix elements

$$
\left\langle\Phi^{N} \mu^{\prime}, \epsilon^{\prime} f, \sigma^{\prime}|K(\mathcal{E})| \Phi^{N} \mu, \epsilon f, \sigma\right\rangle,
$$

and then diagonalize $\{K(\mathscr{E})\}_{\mu, \sigma ; \mu^{\prime}, \sigma^{\prime}}$ according to Eq. (11). One is now able to construct the eigenvectors of the matrix

$$
\left\langle\Phi^{N} \lambda^{\prime}, \epsilon^{\prime} f, \rho^{\prime}|H| \Phi^{N} \lambda, \epsilon f, \rho\right\rangle
$$

according to Eq. (9). We denote these eigenvectors by $\left|\Phi^{N} e \Gamma \mathcal{E}\right\rangle$.

(iii) Obtain the resonance energies. The discrete energies $E_{\beta}$ obtained in (i) are shifted by a secondorder discrete-continuum-discrete interaction represented by the matrix $F_{\beta \beta^{\prime}}(E)$, which must be constructed according to Eq. (20). Diagonalize the matrix $E_{\beta} \delta_{\beta \beta^{\prime}}+F_{\beta \beta^{\prime}}(E)$ as indicated in Eq. (21), and denote the eigenvectors by $\left|\Phi^{N+1} \gamma\right\rangle$ and the eigenvalues by $E_{\gamma}(E)$. Note that these eigenstates and eigenvalues depend on the energy $E$ of the finalstate wave function [Eq. (15)]. Solve Eq. (23) to ob- 
tain the experimentally observable resonance energies. Alternatively, plot $y=E_{\gamma}(E)$ vs $E$; the intersections of this curve with $y=E$ give the experimentally observable resonance energies.

(iv) Determine the coefficients $C_{\Gamma, \Lambda}(E)=\bar{U}_{\Gamma, \Lambda}(E)$ $\cos \pi \varphi_{\Lambda}$. First, construct $\{\bar{K}(E)\}_{\Gamma, \Gamma}$, according to Eq. (31). Second, diagonalize $\{\bar{K}(E)\}_{\Gamma, \Gamma}$, according to $\mathrm{Eq} .(30)$.

(v) Form the augmented discrete states $\left|\Phi^{N+1} \gamma+\right\rangle$ according to Eq. (32).

(vi) Partial cross sections for the $(\mu, \sigma)$ channel are calculated from Eqs. (43) and (46), where the coefficients $\chi_{\Lambda}^{(\mu, \sigma-)}$ are obtained from Eq. (42), Total cross sections are calculated from Eq. (49), using the continuum representation defined in Eq. (48). The total cross section at a resonance energy is calculated from Eq. (55). The partial widths at a resonance energy are calculated from Eq. (56).

With regard to step (i), calculated level separations for the configuration $4 d^{9} 4 f^{N+1}$ tend to be larger than experiment. ${ }^{5}$ This problem, due to the neglect of interaction with highly excited configurations, is well known for other rare-earth spectra ${ }^{19}$ and is usually bypassed by a fitting procedure. ${ }^{5}$ A theoretical justification for the fitting procedure remains to be given. Configuration interaction calculations for level separations are being attempted, but convergence of the calculated level separations to experiment is very slow, since many configurations must be included. ${ }^{20}$ Concerning configuration interaction, it should be noted that the same Slater integrals that split the $4 d^{9} 4 f^{N+1}$ terms by about $20 \mathrm{eV}$ appear in the interaction of the $4 d^{10} 4 f^{N}$ ground configuration with multiply excited configurations such as $4 d^{8} 4 f^{N+2}$. It is known that similar multiply excited configurations (e.g., $4 d^{8} \in f \epsilon^{\prime} f$ ) are important in calculating the $4 d$-shell photoionization cross section in xenon. ${ }^{21}$ However, while interaction of the configurations $4 d^{10} 4 f^{N}$ and $4 d^{9} 4 f^{N+1}$ with highly excited configurations may be accommodated within the framework of this paper, we have not dealt with these problems.

In conclusion, it should be pointed out that the theory presented here should be applicable to other similar experimental data for which the potential barrier plays a crucial role. We particularly have in mind the $3 p$-shell photoabsorption spectra in the transition metals, for which such data exist. ${ }^{22}$ In this case the potential barrier is not as well defined. Nevertheless, the $3 d$ orbit overlaps the $3 p$ orbit, whereas higher $d$ orbits do not. The bulk of the absorption strength is thus concentrated in $3 p \rightarrow 3 d$ transitions. ${ }^{3}$ Other examples exist for which the cross-section formulas derived here should apply. ${ }^{23}$ The partial cross-section formulas would be useful in interpreting the results of future photoelectron spectroscopy experiments. They would also serve as inputs for future photoelectron angular distribution calculations. Also to be noted is that electron scattering experiments using rare-earth or transition-metal targets should give rise to spectra that are similar to those for photoabsorption when the electron's energy is sufficient to excite the $4 d$ or $3 p$ shell.

\section{ACKNOWLEDGMENTS}

I wish to thank Professor U. Fano for suggesting this problem to me, for many valuable discussions, and for constant encouragement. I am also grateful to J. L. Dehmer for helpful conversations.

\section{APPENDIX: SUM RULE FOR DIPOLE TRANSITIONS OF TYPE $l_{i}^{4 l_{i}+2} l_{f}^{N} \rightarrow l_{i}^{4 l_{i}+1} l_{f}^{N+1}$}

We present here a sum rule for transitions from a closed shell to a partially filled shell. Our results may be described simply by application to the case of $4 d$-shell photoabsorption in the trivalent rare earths. We obtain the sum of line strengths ${ }^{24}$ for transitions from a particular level of an initial configuration $4 d^{10} 4 f^{N}$ to all levels of a final configuration $4 d^{9} 4 f^{N+1}$. The summed line strength is shown to be independent of the initial level of the $4 d^{10} 4 f^{N}$ configuration and proportional to the number of vacancies in the $4 f^{N}$ shell (i.e., $14-N$ ). The exclusion principle is thus adduced as the reason for the observed decrease of $4 d$ photoabsorption strength in the rare earths with increasing atomic number. The calculation of the sum rule follows.

We consider transitions from a closed shell to a partially filled shell, i.e., $\left(n_{i} l_{i}\right)^{4 l_{i}+2}\left(n_{f} l_{f}\right)^{N}$ $\rightarrow\left(n_{i} l_{i}\right)^{4 l_{i}+1}\left(n_{f} l_{f}\right)^{N+1}$, where $l_{i}\left(l_{f}\right)$ is the single-particle angular momentum in the initial (final) subshell. The state of the filled subshell $l_{i}^{4} l_{i}+2$ is ${ }^{1} S$, and the state of $l_{f}^{N}$ is identified by the quantum numbers $\alpha L S$, where $\alpha$ denotes any additional quantum number necessary to specify the state uniquely. Similarly, the subshell $l_{i}^{4} l_{i}{ }^{+1}$ is in a doublet $l_{i}$ state, and the state of $l_{f}^{N+1}$ is identified by the quantum numbers $\beta \bar{L} \bar{S}$. All other subshells are assumed closed and hence are ignored. We may write the matrix element of the $q$ th component of the dipole operator as $^{25}$

$$
\begin{aligned}
&\left\langle l_{i}^{4 l_{i+2}}(00) l_{f}^{N}(\alpha L S) L S J M\right| \sum_{j} r_{j} C_{\alpha}^{[1]}\left(\Omega_{j}\right)\left|l_{i}^{4} l_{i+1}\left(l_{i} \frac{1}{2}\right) l_{f}^{N+1}(\beta \bar{L} \bar{S}) L^{\prime} S^{\prime} J^{\prime} M^{\prime}\right\rangle \\
&\left.=\left(n_{i} l_{i}|r| n_{f} l_{f}\right)\left(l_{i}|| C^{[1]}|| l_{f}\right)\left(4 l_{i}+2\right)^{1 / 2}(N+1)^{1 / 2}\left(l_{f}^{N} \alpha L S \mid\right\} l_{f}^{N+1} \beta \bar{L} \bar{S}\right) \\
& \times(-1)^{l_{i}+\bar{L}+L}, \frac{\left[L^{\prime}\right]^{1 / 2}[\bar{L}]^{1 / 2}[\bar{S}]^{1 / 2}}{\left[\frac{1}{2}\right]^{1 / 2}\left[l_{i}\right]^{1 / 2}\left[S^{\prime}\right]^{1 / 2}}\left\{\begin{array}{lll}
l_{f} & \frac{1}{L} & l_{i} \\
L^{\prime} & \bar{L} & L
\end{array}\right\} \delta_{S S^{\prime}}
\end{aligned}
$$




$$
\times(-1)^{L+S-M}\left(\begin{array}{rrr}
J^{\prime} & 1 & J \\
M^{\prime} & q & -M
\end{array}\right) \quad[J]^{1 / 2}\left[J^{\prime}\right]^{1 / 2} \quad\left\{\begin{array}{lll}
1 & J & J^{\prime} \\
S & L^{\prime} & L
\end{array}\right\}
$$

In Eq. (A1), $\left(n_{i} l_{i}|r| n_{f} l_{f}\right)$ is the radial matrix element; $\left(l_{i}|| C^{[1]}|| l_{f}\right)$ is the reduced matrix element of the spherical harmonic $C_{q}^{[1]} ;\left(4 l_{i}+2\right)^{1 / 2}(N+1)^{1 / 2}$ is the product of "weight factors" arising from the $\left(4 l_{i}+2\right)$ equivalent "interacting" electrons in the initial state and the $(N+1)$ equivalent "interacting" electrons in the final state ${ }^{25}$; the next factor is the coefficient of fractional parentage necessary to decouple a single electron from the subshell $l_{f}^{N+1}$; the $6-j$ coefficient

$$
\left\{\begin{array}{lll}
l_{f} & 1 & l_{i} \\
L^{\prime} & \bar{L} & L
\end{array}\right\}
$$

is proportional to the amplitude for recoupling the angular momenta of the system ${ }^{26}$; the $\delta$ function $\delta_{s}$, arises because the dipole operator is spin independent; the $3 j$ symbol

$$
\left(\begin{array}{rrr}
J^{\prime} & 1 & J \\
M^{\prime} & q & -M
\end{array}\right)
$$

is proportional to the amplitude for vector addition of the photon and the total initial angular momentum $J$; the factor

$$
[J]^{1 / 2}\left[J^{\prime}\right]^{1 / 2}\left\{\begin{array}{ccc}
1 & J & J^{\prime} \\
S & L^{\prime} & L
\end{array}\right\}
$$

is, to within a phase, the line strength factor ${ }^{27}$; the factors such as $[J]^{1 / 2} \equiv[2 J+1]^{1 / 2}$ arise primarily because of the use of the more symmetric $3 j$ symbols rather than Clebsch-Gordan coefficients ${ }^{25}$; and, finally, the phase convention used is that of Ref. 25 .

We now wish to sum the line strength over all final-state quantum numbers. For compactness, we specify the configurations by the following symbols:

$$
\begin{aligned}
& \Gamma_{i}(\alpha L S) \equiv l_{i}^{4} l_{i}+2(00) l_{f}^{N}(\alpha L S) L S, \\
& \Gamma_{f}\left(\beta \bar{L} \bar{S}, L^{\prime} S^{\prime}\right) \equiv l_{i}^{4} l_{i} i^{+1}\left(l_{i} \frac{1}{2}\right) l_{f}^{N+1}(\beta \bar{L} \bar{S}) L^{\prime} S^{\prime} .
\end{aligned}
$$

The line strength is then defined as

$$
S\left(\Gamma_{i} J ; \Gamma_{f} J^{\prime}\right) \equiv \sum_{M, q, M^{\prime}}\left|\left\langle\Gamma_{i} J M\left|\sum_{j} r_{j} C_{q}^{[1]}\left(\Omega_{j}\right)\right| \Gamma_{f} J^{\prime} M^{\prime}\right\rangle\right|^{2}
$$

and the sum of $S\left(\Gamma_{i} J ; \Gamma_{f} J^{\prime}\right)$ over all final-state quantum numbers may be reduced to

$$
\left.\sum_{\beta \bar{L} \bar{S}} \sum_{L^{\prime} S^{\prime} J^{\prime}} S\left(\Gamma_{i}(\alpha L S) J ; \Gamma_{f}\left(\beta \bar{L} \bar{S}, L^{\prime} S^{\prime}\right) J^{\prime}\right)=\frac{\left(n_{i} l_{i}|r| n_{f} l_{f}\right)^{2}\left(l_{i}|| C^{[1]}|| l_{f}\right)^{2}}{[1]\left[l_{f}\right][L][S]} \sum_{\beta \bar{L} \bar{S}}[\bar{L}][\bar{S}](N+1)\left(l_{f}^{N} \alpha L S \mid\right\} l_{f}^{N+1} \beta \bar{L} \bar{S}\right)^{2} .
$$

In obtaining Eq. (A4) we used Eqs. (A3) and (A1) as well as standard results ${ }^{28}$ for summations over $3 j$ symbols and $6 j$ coefficients. The sum over the daughter terms in Eq. (A4) is obtained from the relation $^{29}$

$$
\begin{aligned}
\sum_{\beta \bar{L} \bar{S}}[\bar{L}][\bar{S}] & \left.(N+1)\left(l_{f}^{N} \alpha L S \mid\right\} l_{f}^{N+1} \beta \bar{L} \bar{S}\right) \\
\times\left(l_{f}^{N+1} \beta \bar{L} \bar{S}\right. & \left\{\mid l_{f}^{N} \alpha^{\prime} L S\right) \\
& =\delta_{\alpha \alpha^{\prime}}[L][S]\left(4 l_{f}+2-N\right) .
\end{aligned}
$$

Hence, the desired sum rule is

$$
\begin{aligned}
\sum_{\beta \bar{L}} \sum_{\bar{S}} \sum_{L^{\prime} S^{\prime} J^{\prime}} S\left(\Gamma_{i} J ; \Gamma_{f} J^{\prime}\right)=\frac{\left(n_{i} l_{i}|r| n_{f} l_{f}\right)^{2}\left(l_{i}|| C^{[1]}|| l_{f}\right)^{2}}{3\left(2 l_{f}+1\right)} \\
\times\left(4 l_{f}+2-N\right) \cdot \quad(\mathrm{A} 6)
\end{aligned}
$$

The sum rule in Eq. (A6) is independent of the initial-state quantum numbers $L$ and $S$ and is proportional to the number of vacancies $4 l_{f}+2-N$ in the initial partially filled shell. One may also verify that using intermediate coupling for the initial and final states in Eq. (A1) leads to the same result as (A6), with only straightforward modifications of the above procedure. Note that in comparisons of absorption strength for configurations differing in the number of vacancies in the $l_{f}$ subshell the radial matrix elements $\left(n_{i} l_{i}|\gamma| n_{f} l_{f}\right)$ will, in general, differ. Hence, the relation between total absorption strength and the available number of vacancies is not necessarily linear.

Extension of the above sum rule to the general case of $l_{i}^{N} l_{f}^{M} \rightarrow l_{i}^{N-1} l_{f}^{M+1}$ transitions is difficult. While the calculation of the dipole matrix element by the methods of Ref. 25 presents no problem, the summation over final-state quantum numbers is quite complicated because, seemingly, one cannot avoid sums over angular momenta appearing both in $n j$ symbols and in coefficients of fractional parentage.

\footnotetext{
*Work supported by the U.S. Atomic Energy Commission, Contract No. C00-1674-47.

†Presented as part of a thesis to the Department of Physics, The University of Chicago, in partial fulfillment of the requirements for the $\mathrm{Ph} . \mathrm{D}$. degree.

National Science Foundation Graduate Fellow.

$\$_{\text {Present address: Department of Physics, Imperial }}$ College, London S.W. 7, England.

${ }^{1}$ T. M. Zimkina, V. A. Fomichev, S. A. Gribovskii, and I. I. Zhukova, Fiz. Tverd. Tela $\underline{9}, 1447$ (1967); $\underline{9}$,
} 
1490 (1967) [Sov. Phys. Solid State 9, 1128 (1967); 으, 1163 (1967)]; T. M. Zimkina and S. A. Gribovskii, J. Phys. (Paris) (to be published).

${ }^{2}$ R. Haensel, P. Rabe, and B. Sonntag, Solid State Commun. $\underline{8}, 1845(1970)$.

${ }^{3}$ J. L. Dehmer, A. F. Starace, U. Fano, J. Sugar, and J. W. Copper, Phys. Rev. Letters 26, 1521 (1971).

${ }^{4}$ F. Herman and S. Skillman, Atomic Structure Calculations (Prentice-Hall, Englewood Cliffs, N. J., 1963).

${ }^{5} \mathrm{~J}$. Sugar, first following paper, Phys. Rev. B $\underline{5}$, 1785 (1972), Paper II of this series.

${ }^{6}$ U. Fano, Phys. Rev. 124, 1866 (1961).

${ }^{7}$ F. H. Mies, Phys. Rev. 175, 164 (1968).

${ }^{8} \mathrm{~F}$. A. Cotton and G. Wilkinson, Advanced Inorganic Chemistry, 2nd ed. (Interscience, New York, 1966), Chap. 31. See also the tabulated radial wave functions in Ref. 4.

${ }^{9}$ J. L. Dehmer and A. F. Starace, second following paper, Phys. Rev. B $\underline{5}, 1792$ (1972), Paper III in this series.

${ }^{10}$ Tabulated potentials are given, e.g., by Ref. 4 and by T. A. Carlson, C. C. Lu, T. C. Tucker, C. W. Nestor, and F. B. Malik, Oak Ridge National Laboratory Report No. ORNL-4614, UC-34-Physics, 1970 (unpublished).

${ }^{11}$ Photoelectron spectroscopy experiments would determine these energies.

${ }^{12}$ Since the theory of multichannel scattering in the standing-wave representation is dispersed in the literature we go to some length to make this paper self-contained. A unified treatment is given by $\mathrm{U}$. Fano and $\mathrm{F}$. Prats, Proc. Natl. Acad. Sci. (India) A33, 553 (1963). See also U. Fano and J. W. Cooper [Rev. Mod. Phys. 40, 441 (1968), Sec. 6.2] for an excellent comparison between reaction matrix theory and other approaches in collision theory. Less detailed discussions are given in standard references, e.g., L. S. Rodberg and R. M. Thaler, Introduction to the Quantum Theory of Scattering (Academic, New York, 1967), Chap. 9; R. G. Newton, Scattering Theory of Waves and Particles (McGraw-Hill, New York, 1966), pp. 184, 190, and 303; M. L. Goldberger and K. M. Watson, Collision Theory (Wiley, New York, 1964), Sec. 5.6; and N. F. Mott and H. S.W. Massey, The Theory of Atomic Collisions, 3rd ed. (Oxford U. P., London, 1965), p. 372, Chap. XIV, Sec. 1.
${ }^{13} \mathrm{U}$. Fano and J. W. Cooper, Rev. Mod. Phys. 40, 441 (1968), Sec. 2.4. To normalize discrete levels per unit energy one multiplies the discrete wave function by $\left[\left(n^{*}\right)^{3} / 2 I_{H}\right]^{1 / 2}$, where $n^{*}$ is the effective quantum number and $I_{H}$ is the ionization potential for the hydrogen atom.

${ }^{14}$ A. F. Starace, Phys. Rev. A 2 , 118 (1970).

${ }^{15}$ This procedure for obtaining the asymptotic form of the wave function follows the development in Ref. 6 leading up to Eq. (5).

${ }^{16}$ This shift is a standard part of Feshbach's resonance theory, H. Feshbach, Ann. Phys. (N. Y.) 5, 357 (1958).

${ }^{17}$ A. F. Starace, Phys. Rev. A $\underline{3}, 1242$ (1971). Nonlocality is introduced in this problem by our use of an incomplete set of basis sets, namely, those having the configurations $\Phi^{N+1}$ or $\Phi^{N} \epsilon f$.

${ }^{18}$ Reference 6, Eq. (65).

${ }^{19}$ B. G. Wybourne, Spectroscopic Properties of Rare Earths (Interscience, New York, 1965), Secs. 2-18.

${ }^{20} \mathrm{~J}$. C. Morrison and K. Rajnak, Phys. Rev. A $\underline{4}, 536$ (1971); D. J. Newman and C. D. Taylor, J. Phys. B $\underline{4}$, 241 (1971).

${ }^{21}$ M. Ya Amusia, N. A. Cherepkov, and L. V. Chernysheva, Zh. Eksperim.i Teor. Fiz. 60, 160 (1971) [Sov. Phys. JETP 33,90 (1971)].

${ }^{22}$ B. Sonntag, R. Haensel, and C. Kunz, Solid State Commun. 7, 597 (1969).

${ }^{23}$ See, e.g., R. Haensel, K. Radler, B. Sonntag, and C. Kunz, Solid State Commun. 7 , 1495 (1969).

${ }^{24}$ The term "line strength" is defined as the square of the dipole matrix element summed over initial- and finalstate projection quantum numbers. E. U. Condon and G. H. Shortly, The Theory of Atomic Spectra (Cambridge U. P., Cambridge, England, 1935), Sec. 74.

25 The application of Racah algebra and angular momentum diagrams to the evaluation of matrix elements of tensor operators for arbitrary configurations has recently been reviewed by J. S. Briggs, Rev. Mod. Phys. $\underline{43}$, 189 (1971)

${ }^{26} \mathrm{U}$. Fano and G. Racah, Irreducible Tensorial Sets (Academic, New York, 1959), Chaps. 15 and 17.

${ }^{27} \mathrm{~B}$. W. Shore and D. H. Menzel, Principles of Atomic Spectra (Wiley, New York, 1968), Sec. 10.9.

${ }^{28}$ Reference 27, pp. 276 and 283.

${ }^{29}$ G. Racah, Phys. Rev. $\underline{63}, 367$ (1943), Eq. (20). 